\title{
Did we take the right train in promoting the concept of "Neurodevelopmental disorders"?
}

\author{
Bruno Falissard ${ }^{1}$
}

Published online: 18 February 2021

(c) Springer-Verlag GmbH, DE part of Springer Nature 2021

One day I woke up, and I realized, with the same confused astonishment as when you come out of a dream, that neurodevelopmental disorders were everywhere. Of course, the term in itself already existed for years, but here, it was different. In my department, in congresses, in papers, all around the world, people seemed comfortable and even proud to pronounce "neurodevelopmental disorders".

At first, I felt somewhat upset. Child and adolescent psychiatry suffers so much from a lack of recognition that if we consider that a good part of our patients suffers from a neurodevelopmental condition, then it will be obvious for politicians and administrative staff that these patients have to be treated by neurologists and not psychiatrists. Anyway, this was not a big deal after all. No need to be so grouchy. We are used to these palace revolutions: we had psychoanalysis up to the $70 \mathrm{~s}$, then statistics with the DSM, and now, obviously, the "neuro" prefix being everywhere (neuroeconomics, neurophilosophy, etc.); child and adolescent psychiatry has definitely to deal with neurodevelopmental disorders. OK.

In fact no! No, because 2 years ago, a senior manager of the health care system of an important French region decided to transform authoritatively an important part of child and adolescent psychiatric outpatient clinics into diagnostic centers for neurodevelopmental disorders (NDD). If it had happened (and it almost happened), hundreds of thousands of young patients would have been suddenly without any psychiatric care. This unfortunate episode should incite us to think more carefully about the wording we use to define our diseases or disorders. Let us look at it a little more closely across five short propositions.

Bruno Falissard

bruno.falissard@gmail.com

1 CESP, INSERM U1018, Université Paris-Saclay, UVSQ,

Villejuif, AP-HP, France

\section{Proposition 1. The late XXth century and $X X I$ th century saw an ultra-biologization of medicine}

The dazzling emergence of biopharmaceutical products during this period, in particular monoclonal antibodies to treat cancer or inflammatory diseases, is a strong symbol of this phenomenon. MRI, PET-scan, PCR are among numerous acronyms that are now associated with modern occidental medicine. Many sociological publications have discussed this transformation [1].

\section{Proposition 2. Child and adolescent psychiatry followed the same trend-this is why NDD has been so successful}

Historical studies that describe how the concept of NDD emerged are rare. The notion of neurodevelopmental disability seems to have appeared first in the 70s. The objective was twofold: to separate disabilities that affected the body from those affecting behaviors and cognitions, and to set aside the problematic concept of "mental retardation". Neurodevelopmental disability early on was a very broad category that included ADHD, ASD, but also blindness or epilepsy [2].

Scientific articles including the term "Neurodevelopmental disorders" appeared mainly after 2010. A trigger of this rise is perhaps the fifth edition of "Rutter's child and adolescent psychiatry", which was published in 2008. In this famous textbook appeared a chapter entitled: "Neurodevelopmental disorders: conceptual issues". A definition of NDD was proposed:

1. An onset that is invariably during infancy or childhood.

2. An impairment or delay in the development of functions that are strongly related to biological maturation of the central nervous system. 
3. A steady course that does not involve the remissions and relapses that tend to be characteristic of many mental disorders.

Of course, the publication of the DSM-5 in 2013 helped a lot to publicize the concept, which became universally adopted.

\section{Proposition 3. Management and scientific paradigms are not independent}

Between 1990 and 2020, there has been an impressive penetration of the evidence-based medicine paradigm, and more generally of results coming from scientific studies, in the health care system as a whole, and even in the society itself. The "New Public Management", the "What Works" movement have promoted the use of objective indicators, statistics, and randomized experiments in most domains. Systematic reviews and meta-analyses are intensively used to design Evidence-Based Practices [3] or Evidence-Based Policies that all professionals are supposed to follow.

This praiseworthy evolution had noteworthy side effects regarding the way politicians and managers view the desirable evolution of child and adolescent psychiatry. Indeed, if efficiency comes from what is published in high impact factor journals, and if randomized controlled trials (RCTs) and neuroscientific studies are most often acclaimed in these journals, then it makes sense to design mental health care systems according to this body of knowledge. For the best, and the worst.

\section{Proposition 4. Psychiatric diagnoses are more than categories for patients used by a physician, they are also social constructs}

This is a specificity of psychiatry. Lung cancer or multiple sclerosis are medical entities that have more or less the same meaning whether you are a physician or not. This is not the same for depression, schizophrenia, or autism. Mental diseases or disorders fascinate people, and because of that, people integrate them into their day-to-day vocabulary.

It is fascinating to see how the concept of autism has changed in fiction between 1990 and 2010. Dr. Sheldon Cooper (the Big Bang Theory), Dr. Temperance (Bones), or Amélie Poulain are presented as people with an autistic phenotype that is completely different from the character of Raymond Babbitt (Rain man). It seems that artists understood very early (and long before the DSM-5) that autism is a phenotype that is not limited to a severe disability. In these fictions, it is remarkable that autistic people are not considered anymore as psychiatric patients, they are just different, with particularities that can be positive and negative.

\section{Proposition 5. ASD and NDD are two incompatible notions}

ADHD and autism are spectrum disorders; this is now clear. What is not so clear in psychiatric textbooks is that people in the mildest categories of these spectrums have not only difficulties but also positive traits (as do those with more severe forms, of course). Children with ADHD can be spontaneous, creative, with a good sense of humor, while people with ASD rarely lie, are less materialistic and of course, can have terrific cognitive capabilities. In short, people in the mildest category of the ADHD or ASD spectra are just different. The main problem of these persons is that our societies are not compatible with their difference and it can make life difficult for them. So difficult, that they can suffer from a "significant impairment in social, occupational, or other important areas of current functioning" and thus fulfill this criterion used in several DSM disorders.

Now, if we come back to the previous definition of NDD, it requires the existence of "an impairment or delay in the development of functions that are strongly related to biological maturation of the central nervous system".

Does it make sense, is it ethical to claim that a child, with positive and negative characteristics due to his or her difference, has "an impairment [due to the] biological maturation of the CNS"?

\section{Conclusion}

Scientists are free to conceptualize their objects of research and there is no doubt that the construct of NDD is interesting and will help us to make progress in our understanding of some child and adolescent psychiatric disorders [4]. There is, however, a risk. Non-scientists can misuse scientific constructs; this applies particularly to politicians and administrators. Because representations of mental health problems in society are so farfetched, this risk of misuse is particularly important in child and adolescent psychiatry and the disaster in France delineated above almost happened recently because of that.

From a clinical point of view, the most severe forms of ASD, ADHD, intellectual disabilities, or specific learning disorders are indeed compatible with the definition of an NDD. However, this is no more true for the mildest forms of these phenotypes. Psychiatrists and society accept now that autism corresponds to a different way of existing, the intensity of which can vary in important proportions so that the same word "autism" can be used to label very different 
children. For some of them, invoking a problem of "biological maturation of the CNS" raises ethical concerns. In psychiatry, there is a very sad history of pathologizing psychological differences.

In the end, is the NDD concept worth it?

\section{References}

1. Clarke AE, Shim JK, Mamo L, Fosket JR, Fishman JR (2003) Biomedicalization: Technoscientific transformations of health, illness, and U.S. biomedicine. Am Sociol Rev 68(2):161-194
2. Ismail FY, Shapiro BK (2019) What are neurodevelopmental disorders? Curr Opinion Neurol. 32(4):611-616

3. James W. Drisko, Melissa D. Grady (2019) Evidence-based practice in clinical social work. 2e éd. Springer International Publishing (Essential Clinical Social Work Series)

4. Septier M et al (2019) Increased risk of ADHD in families with ASD. Eur Child Adolesc Psychiatry. 28:281-288 